\title{
MeV to TeV Physics with Parity-Violating Electron Scattering
}

\author{
Krishna Kumar*t \\ E-mail: kkumar@physics . umass.edu
}

\begin{abstract}
The technique of measuring parity-violating asymmetries in the scattering of longitudinally polarized electrons off unpolarized fixed targets is well-established and is being applied to a variety of applications to address fundamental questions in nuclear and particle physics. One thrust is the measurements of nucleon neutral weak form factors at intermediate four-momentum transfer $\left(0.1<Q^{2}<1\right)(\mathrm{GeV} / \mathrm{c})^{2}$ which provides information about the role of virtual strange quarks on the charge and current distributions inside nucleons. A new topic is the elastic neutral weak amplitude at very low $Q^{2}$ from scattering off a heavy spinless nucleus, which is sensitive to the presence of a neutron skin. Finally, we discuss the neutral current elastic amplitude at very low $Q^{2}$ off protons and electrons and in the DIS regime off deuterium, which allows precision measurements of the weak mixing angle at low energy and is thus sensitive to new physics at the $\mathrm{TeV}$ scale. The physics implications of recent results, potential measurements from experiments under construction as well as new ideas at future facilities are discussed.
\end{abstract}

50th International Winter Meeting on Nuclear Physics

23-27 January 2012

Bormio, Italy

\footnotetext{
* Speaker.

${ }^{\dagger}$ work is funded in part by US Department of Energy Grant No. DE-FG02-88R40415-A018
} 


\section{Introduction}

The technique of electron scattering has been used for more than fifty years to study the substructure of matter using the cleanliness and firm theoretical underpinnings of electromagnetic interactions. Soon after parity-violation in the weak interactions was first hypothesized and then measured in the late 1950's, Zel'dovich speculated that there might be an analogous parity violating neutral current interaction [1]. He noted that if such an interaction existed, then parity violation would be manifested in lepton-nucleon scattering due to the interference between the weak and electromagnetic amplitudes. He predicted that if one scatters longitudinally polarized electrons off unpolarized protons and flipped the sign of the beam polarization, the fractional difference in the cross-section would be:

$$
A_{\mathrm{PV}} \equiv \frac{\sigma_{R}-\sigma_{L}}{\sigma_{R}+\sigma_{L}} \simeq \frac{\left|A_{Z}\right|}{\left|A_{\gamma}\right|} \simeq \frac{G_{F} Q^{2}}{4 \pi \alpha} \simeq 10^{-4} Q^{2}
$$

For typical fixed target experiments, $A_{P V}$ ranges from roughly $10^{-4}$ to as small as $10^{-7}$. In the mid-seventies, parity violation in deep inelastic electron nucleon scattering was first observed at SLAC [2], from which the electron-quark weak neutral current coupling could be extracted. The measurement was an important test of the $\mathrm{SU}(2) \times \mathrm{U}(1)$ gauge theory of electroweak interactions, and the extracted value of the electroweak mixing angle $\sin ^{2} \theta_{W}$ matched the corresponding value obtained from neutral current neutrino scattering experiments.

Over the past 20 years, the experimental techniques employed to measure these tiny left-right asymmetries have been steadily refined such that statistical and systematic errors better than 1 part per billion (ppb) are possible [3]. Depending on the choice of target and kinematic variables, this has facilitated measurements in several important physics topics, such as many-body nuclear physics, nucleon structure and searches for physics beyond the standard model at the TeV scale. In this review, we discuss the principal physics thrusts of the experimental program, describe the status of ongoing experiments, and speculate on future directions of this research.

\section{Strangeness in Nucleons}

Over the past two decades, several experimental programs have focused on probing for the manifestations of strangeness in nucleon properties, such as mass, spin, momentum, magnetic moment and charge radius. A clean measurement of the contribution of strange quarks to any of these properties would be a dramatic proof of non-trivial dynamics of sea quarks inside nucleons, providing a new window into nonperturbative QCD.

There are some indications that the strange quarks might contribute to the mass (via $\pi$-proton scattering measurements) and the spin (via spin-dependent deep inelastic scattering measurements). While these experiments are sensitive to the strange scalar and axial vector matrix elements, parity violating elastic electron scattering can access vector strangeness matrix elements sensitive to the contribution of strange quarks to nucleon charge and magnetic moment distributions [4, 5].

Elastic electron nucleon electromagnetic scattering is well described by the Dirac and Pauli (or equivalently the Sachs electric and magnetic) form factors. One can introduce equivalent neutral weak form factors that would be accessible in parity violating elastic electron scattering. If one 
assumes the validity of the standard model (weak isospin symmetry) and charge symmetry in strong interactions and that only three flavors are active, then one needs three elastic electroweak electron nucleon amplitudes to achieve flavor separation [6]. Thus, for a given value of $Q^{2}$, if the proton and neutron electromagnetic form factors are well measured, the measurement of the neutral weak form factors at the same value of $Q^{2}$ allows the extraction of the strange form factors.

The exact calculation of the strange form factors from QCD is challenging since it involves nonperturbative dynamics of sea quarks. Various model approaches have been used, such as chiral perturbation theory, quark models, lattice gauge theory, Skyrme models and dispersion relations [7, 8]. More recently, new predictions have become available from lattice calculations [9, 10]. It was determined that, in order to comprehensively probe for non-zero strange quark effects in the vector form factors, measurements over the range $0.1<Q^{2}<1(\mathrm{GeV} / \mathrm{c})^{2}$, as well as forward and backward angle measurements off ${ }^{1} \mathrm{H},{ }^{2} \mathrm{H}$ and ${ }^{4} \mathrm{He}$ targets, are required.

\subsection{Current status and new results}

Four experimental programs have reported $A_{\mathrm{P} V}$ measurements $[11,13,14,12,15]$. All data are mutually consistent. One can combine all experimental data below $Q^{2}=0.2 \mathrm{GeV}^{2}$, which are mutually consistent within quoted uncertainties, to obtain the values $G_{M}^{s}=0.28 \pm 0.20$ and $G_{E}^{s}=-0.006 \pm 0.016$ at $Q^{2}=0.1 \mathrm{GeV}^{2}$.

Until recently, the situation was a bit unclear at $Q^{2} \gtrsim 0.2 \mathrm{GeV}^{2}$. While no single measurement established a statistically significant signal for strange form factors, there were two regions where there are hints of non-zero values after the first round of measurements, one at $Q^{2} \sim 0.25 \mathrm{GeV}^{2}$ and one at $Q^{2} \sim 0.6 \mathrm{GeV}^{2}$, albeit with marginal statistical significance. This was the focus of more recent measurements over the past five years.

Most recently, the HAPPEX experiment has reported a new measurement of the neutral weak form factors with ${ }^{1} \mathrm{H}$ at $Q^{2} \sim 0.62 \mathrm{GeV}^{2}$. With a beam energy $E_{b}$ of $3.48 \mathrm{GeV}$ and a mean scattering angle of $13.7^{\circ}$, the result is $A_{\mathrm{PV}}=(-23.803 \pm 0.778$ (stat) \pm 0.359 (syst) $)$ ppm where the standard model expectation with zero strange form factors is $A_{N S}=-24.062 \pm 0.734 \mathrm{ppm}$ [16]. Comparing $A_{N S}$ to the measured $A_{P V}$, the strange quark contributions are determined to be $G_{E}^{s}+0.517 G_{M}^{2}=$ $0.003 \pm 0.010 \pm 0.004 \pm 0.009$, where the error bars correspond to statistical, systematic, and the $A_{N S}$ uncertainties, respectively.

\subsection{Outlook}

While earlier measurements had suggested non-zero strange form factors, the new HAPPEX results leave little room for strangeness dynamics at low $Q^{2}$, a notion supported recent lattice gauge theory calculations [9, 10]. A4 plans to collect data at backward angles on both ${ }^{1} \mathrm{H}$ and ${ }^{2} \mathrm{H}$ targets at $Q^{2}$ of 0.1 . They have already collected data at $0.6 \mathrm{GeV}^{2}$, and plan to release it soon.

\section{Neutron Skin of a Heavy Nucleus}

In a heavy nucleus such as ${ }^{208} \mathrm{~Pb}$, the fractional difference between neutron radius $R_{n}$ and proton radius $R_{p}$ is believed to be several percent. Analogous to the classic measurement of $R_{p}$ via elastic electron electromagnetic scattering, $R_{n}$ can be measured via elastic electroweak scattering [17]. Experimentally, there is some controversy as to how well $R_{n}$ is known [18]; the best guess 
is $\sim 5 \%$. The parity violating asymmetry in elastic scattering off a heavy spinless nucleus is proportional to the ratio of the neutron to proton form factors since the weak neutral current coupling of protons is much smaller than that of neutrons.

A precise measurement of $R_{n}$ can have impact on nuclear theory, atomic parity violation and neutron star structure. Relativistic mean field models tend to favor larger neutron skins than nonrelativistic models because of a larger symmetry energy. The asymmetry measurement at an optimal value of $Q^{2}$ can pin down $R_{n}$ and help eliminate an entire class of models. Knowledge of $R_{n}$ at the $1 \%$ level can reduce the uncertainty in atomic structure of heavy isotopes that can cloud the interpretation of atomic parity violation measurements that test the Standard Model at the TeV scale [19]. Finally, a precision $R_{n}$ measurement improves our understanding of the equation of state of neutron rich matter, which is important for constraining the structure of neutron stars [20].

\subsection{PREX at Jefferson Lab}

With the improvements made on the control of helicity-correlated beam fluctuations, it became feasible to make a measurement of $A_{\mathrm{PV}}$ in elastic scattering off a ${ }^{208} \mathrm{~Pb}$ target. For a beam energy of $1.05 \mathrm{GeV}$ and a scattering angle of $6^{\circ}$ and $Q^{2} \sim 0.01 \mathrm{GeV}^{2}, A_{P V}$ is of order $0.6 \mathrm{ppm}$. A $3 \%$ measurement of $A_{P V}$ leads to a $1 \%$ measurement of $R_{n}$. A new experimental project known as PREX was approved and carried out a first data run at Jefferson Laboratory in 2010.

Several technical innovations were required to overcome new challenges that resulted from the aggressive parameters of the experimental measurement. The experiment ran for about 10 weeks in the spring of 2010. One important new issue was the amount of radiation in the Hall during production data collection. In particular, the vacuum system just downstream of the target was compromised due to radiation and severely affected the efficiency of data collection. At the end of the run, statistics for a $9 \% A_{P V}$ measurement and a $3 \% R_{n}$ determination was accumulated, and this result was recently published [21]. After corrections for beam fluctuations, the grand average was found to be $594 \pm 50$ parts per billion (ppb). After normalizing to the beam polarization and subtracting background, the PREX result is $A_{P V}=656 \pm 60$ (stat) \pm 14 (syst) ppb.

The measured result corresponds to a value for the neutron skin of $R_{n}-R_{p}=+0.33_{-0.18}^{+0.16} \mathrm{fm}$. While the result demonstrates that the neutron RMS radius is $2 \sigma$ larger than that of the protons the result is not yet precise enough to discriminate between various models based on mean field theory and other observables.

\subsection{Outlook for Future Measurements}

The motivation to obtain a robust and model-independent determination of $R_{n}$ over a range of nuclei will remain compelling for years to come. A followup proposal to obtain the remaining statistics required for a $1 \% R_{n}$ measurement by the PREX collaboration has been approved and will likely run in 2014 at Jefferson Laboratory. Two other attractive nuclei to explore $R_{n}$ measurements are ${ }^{48} \mathrm{Ca}$ and ${ }^{120 / 124} \mathrm{Sn}$. In general low $Z$ nuclei tend to have a higher figure of merit due to the fact that the optimum $Q^{2}$ at which one must make the $A_{P V}$ measurement tends to increase, and the figure of merit rises with the square of the asymmetry times the count rate [22].

${ }^{48} \mathrm{Ca}$ is particularly interesting because microscopic calculations may soon be feasible, which would allow $R_{n}$ to be related to poorly studied 3-neutron forces. Sn isotopes are also interesting, 
since many of them have been used in heavy ion collisions to probe the density dependence of the symmetry energy, which has been shown to be related to $R_{n}$. It turns out that ${ }^{48} \mathrm{Ca}$ is an ideal measurement at Jefferson Laboratory with a similar configuration to that used for PREX, except that the beam energy would be raised to $2.2 \mathrm{GeV}$. This is one of the beam energies that would be possible to run simultaneously with a $11 \mathrm{GeV}$ beam in another Hall.

It is interesting to consider measurements of $\mathrm{Pb}$ and $\mathrm{Sn}$ at Mainz in the future, adapting the apparatus that would be required for a high precision proton weak charge measurement (see Sec. 4.2.2). The loss in rate due to the lower beam energy is roughly compensated by the larger available solid angle. The momentum resolution that would be required is about $1 \%$, and a system of baffles could be used to isolate elastic events while rejecting background from inelastics as well as from neutrals [23]. It will be very important to verify measurements of the same isotope in two different laboratories and also obtain consistent results on $R_{n}$ from three different isotopes.

\section{Precision Weak Neutral Current Measurements}

The electroweak interaction has been tested to high precision at high energy colliders, especially by measurements of the properties of the $\mathrm{W}$ and $\mathrm{Z}$ bosons and their couplings to leptons and quarks. While all data are consistent with the standard model, experiments continue to probe for the indirect effects of new physics at the $\mathrm{TeV}$ scale by making more and more precise measurements of electroweak parameters. Weak neutral current (WNC) interactions at $Q^{2} \ll M_{Z}^{2}$ can probe for heavy $Z^{\prime}$ bosons or leptoquarks whose effects might be highly suppressed in measurements on the $\mathrm{Z}$ pole [24]. Since the neutral current amplitude at the $\mathrm{Z}$ pole is imaginary, there are no interference terms with new, real amplitudes. At low $Q^{2}$ on the other hand, interference effects might be measurable if sufficient accuracy is achieved [25].

A general, model-independent way to parametrize the contributions of contact interactions of high-mass particles to low-energy measurements of electron scattering off target fermions $f$ is to use the Lagrangian [26]

$$
\mathscr{L}_{e f}=\sum_{i, j=L, R} \frac{g_{i j}^{2}}{\Lambda^{2}} \bar{e}_{i} \gamma_{\mu} e_{i} \bar{f}_{j} \gamma^{\mu} f_{j}
$$

where $e_{L / R}=\frac{1}{2}\left(1 \mp \gamma_{5}\right) \psi_{e}$ and $f_{L / R}=\frac{1}{2}\left(1 \mp \gamma_{5}\right) \psi_{f}$ are the chirality projections of the fermion spinors, the $g_{i j}$ are the coupling constants, and $\Lambda$ is the mass scale. The goal of low energy neutral current measurements is to reach sufficient sensitivity to access $\Lambda / g_{i j} \geq 1 \mathrm{TeV}$ for as many different initial and final state fermions as possible, similar sensitivity to that of the highest energy colliders.

Three measurement techniques have reached such sensitivity. The weak charge measurement in ${ }^{133} C s$ [27], the NuTeV neutrino deep-inelastic scattering measurement [28] and the measurement of $A_{\mathrm{PV}}$ in electron-electron (Møller) scattering [29] at SLAC. These measurements are used to set important new constraints on new parity-violating contact interactions at the $10 \mathrm{TeV}$ scale.

As we await the onset of data from the Large Hadron Collider (LHC), improving these constraints and expanding their reach to include more fermion scattering combinations takes on increased significance. This is because such measurements will help narrow down the physics mechanisms that are responsible for any observed anomalies at the LHC. In the following, we describe ongoing and future measurements that could improve on the abovementioned three measurements, 
using parity-violating elastic electron-proton and electron-electron scattering, and deep-inelastic electron-deuteron scattering.

\subsection{Lepton-Quark Weak Neutral Current Interactions}

The interactions of the Z-boson at low $Q^{2}$ can be approximated by four-fermion contact interactions. The parity-violating part of the electron-hadron interaction can then be given in terms of phenomenological couplings $C_{i j}$

$$
\mathscr{L}^{P V}=\frac{G_{F}}{\sqrt{2}}\left[\bar{e} \gamma^{\mu} \gamma_{5} e\left(C_{1 u} \bar{u} \gamma_{\mu} u+C_{1 d} \bar{d} \gamma_{\mu} d\right)+\bar{e} \gamma^{\mu} e\left(C_{2 u} \bar{u} \gamma_{\mu} \gamma_{5} u+C_{2 d} \bar{d} \gamma_{\mu} \gamma_{5} d\right)\right]
$$

with additional terms as required for the heavy quarks. Here $C_{1 j}\left(C_{2 j}\right)$ gives the vector (axialvector) coupling to the $j^{\text {th }}$ quark. In the Standard Model, all four couplings are functions of a single parameter: the weak mixing angle $\sin ^{2} \theta_{W}$. The atomic Cesium weak charge measurement measured one combination of $C_{1 u}$ and $C_{1 d}$ precisely.

\subsection{Parity-Violating Elastic Electron-Proton Scattering}

At sufficiently forward angles and low $Q^{2}$, the hadronic structure uncertainty in the WNC elastic electron-proton amplitude becomes small enough such that one can measure the underlying coherent $2 u+d$ e-q amplitude combination to high precision, thus precisely constraining $2 C_{1 u}+$ $C_{1 d}$. This combination is proportional to $1-4 \sin ^{2} \theta_{W}$.

\subsubsection{Qweak at Jefferson Lab}

A $4 \%$ measurement of $A_{P V}$ would achieve a precision of $\delta\left(\sin ^{2} \theta_{W}\right)=0.0007$. This is the goal of the Qweak experiment [30] in Hall C at Jefferson Lab, which was designed over the past few years. Construction of the experiment was completed in Summer 2010, and the first commissioning took place soon after. The target worked successfully at full power and the integrated response of the quartz detectors shows the full statistics of about $6 \mathrm{GHz}$, which means that the dominant sources of noise from electronics and backgrounds are well below the fluctuations due to counting statistics. As of April 2012, the experiment is running well and it is on track to obtain the full statistics before the Jefferson Laboratory summer shutdown in June 2012.

\subsubsection{Potential Future Measurement at Mainz}

It would be highly complementary to obtain a new measurement of the proton weak charge with a new apparatus where the beam energy is smaller than $200 \mathrm{MeV}$. The dominant theoretical uncertainty in the prediction of $A_{P V}$ is due to higher-order radiative corrections involving $\gamma-Z$ box diagrams. Indeed, the size of the correction is roughly proportional to the incident beam energy, so that the systematic error from the correction would be significantly smaller at lower energy. In order to compensate for the lower energy, it will be necessary to obtain data over the full range of the azimuth using a solenoidal spectrometer [23]. Such an experiment would be a flagship measurement of the proposed new MESA facility at Mainz. A measurement of $A_{P V}$ with an ultimate goal of 2 or $2.5 \%$ will remain a compelling case for many years to come. 


\subsection{Parity-Violating Deep Inelastic Scattering}

The upgrade of Jefferson Laboratory to $11 \mathrm{GeV}$ will allow precision measurements in parityviolating deep inelastic scattering (PV DIS). One measurement has publishable results using a 6 $\mathrm{GeV}$ beam and the existing high resolution spectrometers in Hall A [31]. Another measurement has been proposed to use the $11 \mathrm{GeV}$ beam and the upgraded spectrometer system in Hall C [32]. Here we focus on a new dedicated high luminosity apparatus centered around a large superconducting solenoidal magnet [33]. This project, known as SoLID (Solenoidal Large Intensity Device), would allow $\sim 1 \%$ measurements of $A_{P V}$ for the first time over a range of $x$ and $Q^{2}$ values, as high as $x \sim 0.7$ ( $x$ is the fraction of the nucleon momentum carried by the struck quark).

As we describe in the following, PV DIS measurements with such accuracy and kinematic range provides access to novel aspects of nucleon structure, such as charge symmetry violation and investigation of higher-twist effects. Most importantly, PV DIS allows the isolation of the linear combination $2 C_{2 u}+C_{2 d}$, which is difficult to measure using elastic scattering.

\subsection{PVDIS Phenomenology}

$A_{P V}$ in DIS can be written as

$$
\begin{aligned}
A_{P V}= & Q^{2} \frac{G_{F}}{2 \sqrt{2} \pi \alpha}\left[a(x)+\frac{1-(1-y)^{2}}{1+(1-y)^{2}} b(x)\right], \\
& a(x) \equiv \Sigma_{i} f_{i}(x) C_{1 i} q_{i} / \Sigma_{i} f_{i}(x) q_{i}^{2} \\
& b(x) \equiv \Sigma_{i} f_{i}(x) C_{2 i} q_{i} / \Sigma_{i} f_{i}(x) q_{i}^{2} .
\end{aligned}
$$

Here, $f_{i}(x)$ are parton distribution functions and $q_{i}$ are the electromagnetic charges. The $a(x)$ term arises from the product of the electron axial-vector coupling and the quark vector coupling and is typically the dominant term. For an isoscalar target such as deuterium, the dependence on structure largely cancels out in the $A_{P V}$ ratio of the weak and electromagnetic amplitudes:

$$
a(x)=\frac{6}{5}\left[\left(C_{1 u}-\frac{1}{2} C_{1 d}\right)+\cdots\right] ; \quad b(x)=\frac{6}{5}\left[\left(C_{2 u}-\frac{1}{2} C_{2 d}\right) \frac{q(x)-\bar{q}(x)}{q(x)+\bar{q}(x)}+\cdots\right]
$$

where $q(x)=u(x)+d(x)$.

\subsubsection{Charge Symmetry Violation}

From Eqn. 4.5 one obtains $a(x) \approx 1.15$ for an isoscalar target, independent of $x$. This results from the assumption of charge symmetry, where the $u$-quark distribution in the proton is the same as the $d$-quark distribution in the neutron, with a similar assumption for the proton $d$-quark distribution: $u^{p}=d^{n}$ and $d^{p}=u^{n}$. If $a(x)$ can be measured with high precision over a range of $x$ values, one is thus quite sensitive to charge symmetry violation (CSV). If one defines CSV parameters:

$$
\delta u(x)=u^{p}(x)-d^{n}(x) ; \quad \delta d(x)=d^{p}(x)-u^{n}(x),
$$

then dependence of the parity-violating asymmetry for an isoscalar target on CSV is:

$$
\frac{\delta A_{P V}}{A_{P V}}=0.28 \frac{\delta u-\delta d}{u+d} \equiv 0.28 R_{C S V} .
$$


While $R_{C S V}$ is known to be less than 0.01 for $x<0.4$ from neutrino DIS measurements [34], a bag model calculation suggests that $R_{C S V} \sim 0.01$ for $x \sim 0.4$ and rising to 0.02 for $x \sim 0.6$. At high $x$, knowledge of $u+d$ is limited. As $x \rightarrow 1$, if $u+d$ falls off more rapidly than $\delta u-\delta d$, then $R_{C S V}$ might rise to 0.1 at $x \sim 0.7$, which would be observable with a $1 \% A_{P V}$ measurement. Further, $R_{C S V}$ is quite unconstrained at large $x$. There is the tantalizing possiblity that $R_{C S V}$ in the moderate and high $x$ region is a factor of 3 bigger than above-mentioned values, which would be large enough to explain the discrepancy in the neutrino-nucleon DIS measurement (NuTeV anomaly) [34].

\subsubsection{Constraints on New Contact Interactions}

The measurement of the PV DIS asymmetry to an accuracy of $0.6 \%$ at $Q^{2} \sim 5 \mathrm{GeV}^{2}$ and $x \sim 0.4$ for an isoscalar target such as deuterium, coupled with precise knowledge the parameter $a(x)$ from other experiments, the parameter $b(x)$ can be extracted with high accuracy for the first time. This measurement would be robust only if the comprehensive PV DIS program described above is carried out. Indeed, as can be seen from Eqn. 4.5, $a(x)$ is independent of $x$ and simply a function of $\sin ^{2} \theta_{W}$ under the assumption of charge symmetry and assuming that higher twist effects are either directly measured or constrained. The quantity $b(x)$ is also virtually free of structure function uncertainties at high $x$.

The measurement is interesting for several reasons. Firstly, it would test the WNC amplitude in the lepton-quark sector, where there is currently a discrepancy with the theoretical prediction in the $\mathrm{NuTeV}$ result. Secondly, combined with other measurements in elastic electron-proton scattering, precise constraints would be possible on the lesser known axial-vector quark couplings $C_{2 i}$. This would, among other things, provide complementary constraints on various models with new heavy leptophobic $Z^{\prime}$ bosons [35] and leptoquarks [24].

More generally, the projected result for $A_{P V}^{D}$ of $0.6 \%$ translates into a measurement of a specific linear combination of the phenomenological couplings $C_{i j}$ to an accuracy of \pm 0.0083 , more than a factor of 30 better than the best current constraints. This translates into contact interaction scale reach $\gtrsim 2.5 \mathrm{TeV}$. For example, models of lepton compositeness are characterized by strong coupling dynamics. Taking $\sqrt{\left|g_{R R}^{2}-g_{L L}^{2}\right|}=2 \pi$ shows that mass scales as large as $\Lambda=15.5 \mathrm{TeV}$ can be probed, corresponding to electron and quark substructure at the level of $\sim 10^{-20} \mathrm{~m}$.

\subsubsection{The SOLID Proposal}

To comprehensively address the physics topics discussed above experimentally, a series of $A_{P V}$ measurements in the range of 1 to $2 \%$ accuracy are required for $0.3 \lesssim x_{\mathrm{bj}} \lesssim 0.7$, with a lever arm of a factor of 2 in $Q^{2}$ while keeping $W_{\min }^{2}>4$ and $Q_{\min }^{2}>1$. With the upgrade of Jlab, high luminosity with a beam energy of $11 \mathrm{GeV}$ becomes possible. However, to achieve sufficient statistics at the highest possible $Q^{2}$, a spectrometer with at least $50 \%$ acceptance in the azimuth is required.

A large magnet is required to shield the detectors from target photons and to sweep out low energy charged particles. A large volume solenoid would be ideal for the required geometry, mainly the long target length and the large laboratory scattering angles. It turns out that the central tracking magnetic field of one of the collider experiments BaBar, CDF or CLEO would be ideal for this purpose. A proposal for this comprehensive program was approved in January 2010 at Jefferson Laboratory and detailed simulations have been carried out for the case of using the CLEO-II mag- 
net [33]. A significant R\&D program has been launched to develop a detailed experimental design and the project will seek funding in a few years to run at Jefferson Laboratory.

\subsection{Parity-Violating Møller Scattering}

The Feynman diagrams for Møller scattering involve both direct and exchange diagrams that interfere with each other. $A_{P V}$ is given by [36]

$$
A_{P V}=m E \frac{G_{F}}{\sqrt{2} \pi \alpha} \frac{4 \sin ^{2} \theta}{\left(3+\cos ^{2} \theta\right)^{2}} Q_{W}^{e}=m E \frac{G_{F}}{\sqrt{2} \pi \alpha} \frac{2 y(1-y)}{1+y^{4}+(1-y)^{4}} Q_{W}^{e}
$$

where $\alpha$ is the fine structure constant, $E$ is the incident beam energy, $m$ is the electron mass, $\theta$ is the scattering angle in the center of mass frame, $y \equiv 1-E^{\prime} / E, E^{\prime}$ is the energy of one of the scattered electrons, and $Q_{W}^{e}$ (proportional to the product of the electron's vector and axial-vector couplings to the $Z^{0}$ ) is the electron's weak charge.

The electroweak theory prediction at tree level in terms of the weak mixing angle is $Q_{W}^{e}=$ $1-4 \sin ^{2} \theta_{W}$; this is modified at the 1-loop level [37, 38] and becomes dependent on the energy scale at which the measurement is carried out, i.e. $\sin ^{2} \theta_{W}$ "runs". It increases by $\sim 3 \%$ compared to its value at the scale of the $Z^{0}$ boson mass, $M_{Z}$.

\subsubsection{SLAC E158}

The SLAC E158 experiment carried out the first measurement of parity violation in Møller scattering [29]. The experiment was constructed between 2000 and 2002 and final data collection completed in late 2003. The grand average result for the parity-violating asymmetry in Møller scattering at $Q^{2}=0.03 \mathrm{GeV}^{2}$ was found to be: $A_{\mathrm{PV}}=-131 \pm 14$ (stat) \pm 10 (syst) (ppb). This can be compared to the theoretical prediction within the electroweak framework to put limits on new contact interactions at the $\mathrm{TeV}$ scale. From the measured result, and assuming that the only contributing chiral structure comes from the $\Lambda_{\mathrm{LL}}$ term, the $95 \%$ C.L. limit is $7 \mathrm{TeV}$ or $16 \mathrm{TeV}$ depending on the sign of the contact interaction term.

\subsubsection{The MOLLER Experiment at Jefferson Laboratory}

There is strong motivation to make further improvements in the accuracy of weak neutral current coupling constants at low energy. Improved measurements would keep pace with the improved sensitivity for discovery at the multi-TeV scale by experiments at the Large Hadron Collider. With the upgrade of Jefferson Laboratory to $12 \mathrm{GeV}$, a new project called MOLLER (Measurement of Lepton-Lepton Electroweak Reaction) [39] is being designed to improve on the SLAC E158 measurement of the weak charge of the electron $Q_{W}^{e}$ by a factor of five.

Using $\alpha_{\mathrm{EM}}, G_{F}, M_{Z}$ and $m_{t}$ as input, precision measurements of electroweak parameters such $m_{W}$ and $\sin ^{2} \theta_{W}$ can be used to test the electroweak theory at the level of electroweak radiative corrections. Consistency (or lack thereof) of various precision measurements can then be used to constrain $m_{H}$ and search for indications of physics beyond the Standard Model. While there is no significant conflict between such indirect limits of $m_{H}$ with direct collider searches, it is instructive to look at the most significant indirect measurements in detail.

In particular, the $m_{H}$ constraints from the two most precise single measurements of $\sin ^{2} \theta_{W}$ lead to very different conclusions [40]. On the one hand, the left-right asymmetry measurement in 
$\mathrm{Z}$ production from the SLAC Linear Collider $A_{\mathrm{LR}}(\mathrm{had})$ indicates a very small $m_{H}$ which is already ruled out by direct searches. On the other hand, the forward-backward asymmetry measurement in $\mathrm{Z}$ decays to b-quarks from CERN $A_{\mathrm{FB}}(b)[\mathrm{LEP}]$ indicates a very large $m_{H}$ and is in tension with constraints from all indirect measurements. The proposed MOLLER measurement would provide a third independent measurement of $\sin ^{2} \theta_{W}$ with comparable precision.

Further, since MOLLER would be carried out a low energy scale in contrast to the two collider measurements, there is greatly enhanced sensitivity to as yet undiscovered superweak interactions at the $\mathrm{TeV}$ scale. For the $2.3 \%$ total uncertainty envisioned, the sensitivity can be expressed as:

$$
\frac{\Lambda}{\sqrt{\left|g_{R R}^{2}-g_{L L}^{2}\right|}}=\frac{1}{\sqrt{\sqrt{2} G_{F}\left|\Delta Q_{W}^{e}\right|}} \simeq \frac{246.22 \mathrm{GeV}}{\sqrt{0.023 Q_{W}^{e}}}=7.5 \mathrm{TeV}
$$

For example, models of lepton compositeness are characterized by strong coupling dynamics. Taking $\sqrt{\left|g_{R R}^{2}-g_{L L}^{2}\right|}=2 \pi$ shows that mass scales as large as $\Lambda=47 \mathrm{TeV}$ can be probed, far beyond the center of mass energies of any current or planned high energy accelerator. This allows electron substructure to be studied down to the level of $4 \times 10^{-21} \mathrm{~m}$. The strongest constraints on the coefficients in Eqn. (??) come from LEP 2, approaching $5 \mathrm{TeV}$ for specific chiral combinations. However, the parity-conserving cross-sections and forward-backward asymmetries studied at LEP 2 are blind to the parity-violating combination $g_{R R}^{2}-g_{L L}^{2}$ probed by MOLLER.

Using Eqn. (4.9), it is also straightforward to examine its reach in specific models [24]. The sensitivity to specific R-Parity-violating SUSY interactions would be greatly improved [41]. In addition, the MOLLER measurement would provide unique new constraints on combinations of left- and right-handed leptonic couplings to new neutral gauge bosons between 1 and $2 \mathrm{TeV}$ [42], complementing LHC measurements [43]. Finally, the sensitivity to lepton-number violating interactions mediated by doubly charged scalars would be greatly extended.

The MOLLER design shares many similarities to the E158 apparatus described above. The prediction for $A_{P V}$ is 35.6 parts per billion (ppb). The goal is to measure this quantity to a statistical precision of $0.74 \mathrm{ppb}$ and keep systematic errors to be significantly smaller, in order to achieve a $2.3 \%$ measurement of $Q_{W}^{e}$. MOLLER will greatly benefit from the steady improvement in the techniques employed to measure parity-violating asymmetries to sub-ppb systematic precision and to also achieve normalization control at the sub-\% level. Several methods to reverse the sign of the asymmetry will also be employed periodically.

The MOLLER collaboration, a group of $\sim 100$ authors, submitted a proposal to the Jefferson Laboratory Program Advisory Committee in December 2008. The project was approved with strong endorsement. The collaboration is currently formulating an R\& D plan to develop the technical design of the apparatus. The MOLLER collaboration is in the process of seeking project funding of order $20 \mathrm{M}$ from DoE, NSF and international funding agencies. The goal is to launch MOLLER with significant R\&D funding by late 2012, so that construction of the apparatus can begin in late 2014. The MOLLER experiment could then be constructed by 2016, soon after full luminosity beams become available at Jefferson Laboratory.

\section{Summary}

Parity-violating electron scattering is a mature field and addresses fundamental questions in 
a variety of different topics. The E158 experiment at SLAC has produced the most precise measurement of the weak mixing angle at low energy. The Qweak experiment at Jefferson Laboratory is now taking data and is on track to make a weak mixing angle measurement that will be better than the E158 result. The two future proposals at Jefferson Laboratory, SoLID and MOLLER, will lead to important new insights on the structure of the nucleon and yield new and more precise measurements of the weak mixing angle and constitute flagship projects after the $12 \mathrm{GeV}$ upgrade. It will be greatly beneficial to design and carry out a new measurement of the proton weak charge at Mainz that will improve on the Qweak experiment by a factor of 2 .

In parallel, it is greatly motivated to carry out new measurements of $A_{P V}$ on heavy nuclei to measure the ground-state neutron distribution. The PREX measurement is on-going and should produce a $1 \%$ measurement of the neutron radius in ${ }^{208} \mathrm{~Pb}$ in a few years. In the long run, it will be very important to produce comparable measurements using $\mathrm{Ca}$ and $\mathrm{Sn}$ isotopes at Jefferson Laboratory and at Mainz. Finally, the proposed new MESA facility at Mainz has the potential to add important new measurements to the impressive list of parity-violation experiments in order to probe nuclear and nucleon structure and to search for physics beyond the Standard Model.

\section{Acknowledgements}

It is a pleasure to thank the organizers for a stimulating meeting. The contributions of all the experimental collaborations discussed in this review are gratefully acknowledged.

\section{References}

[1] Zel'dovich,Ya.B., J.Exptl.Theoret.Phys. (U.S.S.R.), 36, 1959, pp. 964-966.

[2] Prescott,C.Y., et.al., Phys.Lett., B84, 1979, 524.

[3] Kumar,K.S. and Souder,P.A., Prog.Part.Nucl.Phys., 45, 2000, pp. S333-S395.

[4] Kaplan,D.B. and Manohar,A., Nucl.Phys., B310, 1988, 527.

[5] McKeown,R.D., Phys.Lett., B219, 1989, 140.

[6] Beck,D.H., Phys. Rev., D39, 1989, 3248.

[7] Musolf,M.J., et.al., Phys. Rep., 239, 1994, 1, and references therein.

[8] Beck,D.H. and Holstein,B.R., Int.J.Mod.Phys.,E10, 2001, pp. 1-41.

[9] Leinweber,D.B., et.al, Phys.Rev.Lett. 94, 2005, 212001; Leinweber,D.B., et.al, Phys.Rev.Lett. 97, 2006, 022001.

[10] Doi,T., et.al, Phys. Rev. D80, 094503 (2009). [arXiv:0903.3232 [hep-ph]].

[11] Spayde,D., et.al., Phys.Rev.Lett., 84, 2000, 1106; Hasty,R., et.al., Science, 290, 2000, 2117.

[12] Aniol,K.A., et.al., Phys.Lett., B509, 2001, pp. 211-216.

[13] Maas,F.E. et.al, Phys.Rev.Lett., 93, 2004, 022002; Maas,F.E. et.al, Phys.Rev.Lett., 94, 2005, 152001.

[14] Armstrong,D.S., et.al, Phys.Rev.Lett. 95, 2005, 092001.

[15] Aniol,K.A., et.al, Phys.Rev.Lett. 96, 2006, 022003; Aniol,K.A., et.al, Phys.Lett., B635, 2006, 275. 
[16] Ahmed,Z., et.al, Phys.Rev.Lett. 108, 2012, 102001.

[17] Donnelly,T.W., Dubach,J. and Sick,I., Nucl.Phys., A503, 1989, 589.

[18] Horowitz,C.J., et.al., Phys.Rev., C63, 2001, 025501.

[19] Pollock,S.J., Fortson,E.N. and Wilets,L., Phys.Rev., C46, 1992, 2587.

[20] Horowitz,C.J. and Piekarweicz,J., Phys.Rev.Lett., 86, 2001, 5647.

[21] Abrahamyan,S., et.al, Phys.Rev.Lett. 108, 2012, 112502.

[22] Ban,S, Horowitz,C.J. and Michaels,R., [arXiv:1010.3246 [nucl-th]].

[23] Souder,P.A. and Holmes,R., In *Pasadena 1990, Proceedings, Parity violation in electron scattering* 137-151.

[24] M.J. Ramsey-Musolf, Phys.Rev., C60 (1999) 015501.

[25] K.S. Kumar et.al., Mod.Phys.Lett., A10 (1995) 2979.

[26] E. Eichten, K. D. Lane and M. E. Peskin, Phys. Rev. Lett. 50 (1983) 811.

[27] C.S. Wood et.al., Science, 275 (1997) 1759.

[28] G.P. Zeller et.al., Phys.Rev.Lett., 88 (2002) 091802.

[29] P.L. Anthony et.al, Phys.Rev.Lett., 95 (2005) 081601.

[30] The Qweak Experiment, http://www.jlab.org/qweak/

[31] Jefferson Lab Experiment E05-007, X. Zheng, Contact Person

[32] Jefferson Lab Proposal PR12-07-102, P. Reimer, Contact Person

[33] The SoLID Experiment, Jefferson Lab Proposal PR-09-012, P.A. Souder, Contact Person

[34] J.T. Londergan and A.W. Thomas, hep-ph/0407247 (2004), and references therein.

[35] M.R. Buckley and M.J. Ramsey-Musolf, arXiv:1203.1102[hep-ph]

[36] E. Derman and W. J. Marciano, Annals Phys. 121 (1979) 147.

[37] A. Czarnecki and W. J. Marciano, Phys. Rev. D 53 (1996) 1066.

[38] J. Erler and M. J. Ramsey-Musolf, Phys. Rev. D72 (2005) 073003.

[39] The MOLLER experiment, Jefferson Lab Proposal PR-09-005, K.S. Kumar Contact Person

[40] W. J. Marciano, AIP Conf. Proc. 870 (2006) 236.

[41] M. J. Ramsey-Musolf and S. Su, Phys. Rept. 456 (2008) 1. [arXiv:hep-ph/0612057].

[42] J. Erler and P. Langacker, Phys. Lett. B456 (1999) 68.

[43] Li,Y., Petriello,F. and Quackenbush,S., Phys. Rev. D80, 055018 (2009). [arXiv:0906.4132 [hep-ph]].

[44] A. Kurylov, M.J. Ramsey-Musolf and S. Su, Phys. Rev. D68, (2003) 035008. 